\title{
Probing the transverse single spin asymmetry in the inelastic $J / \Psi$ photoproduction at hadronic colliders
}

\author{
Victor P. Goncalves* \\ High and Medium Energy Group, Instituto de Física e Matemática \\ Universidade Federal de Pelotas \\ Caixa Postal 354, 96010-900, Pelotas, RS, Brazil. \\ E-mail:barroseufpel .edu.br
}

\begin{abstract}
In this contribution we investigate the transverse single spin asymmetry in the inelastic $J / \Psi$ photoproduction in $p^{\uparrow} p$ and $p^{\uparrow} A$ collisions at RHIC energies. At leading order this process probes the gluon Sivers function. We predict large values for the cross sections, which indicates that its experimental analysis is, in principle, feasible. The rapidity dependence of the single spin asymmetry is presented. We obtain that the asymmetry is strongly dependent on the model used for the gluon Sivers function and that it can be probed by the analysis of the $J / \Psi$ production at forward rapidities. Our results indicate that a future experimental analysis of this process can be useful to constrain the gluon Sivers function.
\end{abstract}

XXVI International Workshop on Deep-Inelastic Scattering and Related Subjects (DIS2018)

16-20 April 2018

Kobe, Japan

${ }^{*}$ Speaker. 


\section{Introduction}

The study of high energy processes involving polarized hadrons allows to improve our understanding of the polarized quark and gluon structure of the hadrons and the QCD dynamics at a high - energy scale (See e.g. Refs. [1,2]). In particular, the analysis of the transverse spin phenomena in hard processes is expected to provide a three - dimensional picture of the partons inside the nucleon. One of the current challenges in hadronic physics is the understanding of the large transverse single - spin asymmetries (SSAs), which have been observed in several experiments. A possible explanation for the presence of this asymmetry was proposed many years ago [3] and is known as Sivers effect, which considers the correlation between the transverse momentum of partons and the polarization vector of the nucleon. One important open question is the size of the gluon Sivers function, with no hard constraint existing apart from the positivity bound [4]. In this contribution, we summarize the results presented in Ref. [5], where we have proposed to study the gluon Sivers function in the photoproduction of vector mesons in $p^{\uparrow} p$ and $p^{\uparrow} A$ collisions at high energies.

\section{Formalism}

The basic idea in photon - induced interactions is that an ultra relativistic charged hadron (proton or nucleus) gives rise to strong electromagnetic fields, such that the photon stemming from the electromagnetic field of one of the two colliding hadrons can interact with one photon of the other hadron (photon - photon process) or can interact directly with the other hadron (photon hadron process) [6]. In the particular case of the inelastic $J / \Psi$ photoproduction in $p^{\uparrow} p$ and $p^{\uparrow} A$ collisions, we will assume that the unpolarized hadron ( $p$ or $A$ ) is the source of photons, which interact with the transversely polarized protons at high energies, producing a $J / \Psi$ and dissociating the proton target. In the nuclear case, such approximation is justified due to enhancement by a factor $Z^{2}$ in the nuclear photon flux in comparison to that for a proton, which implies that the photon induced interactions are dominated by photons from the nucleus. In the case of $p^{\uparrow} p$ collisions, the process of interest can be separated by tagging the unpolarized proton in the final state, which is present when it emits the photon. As a consequence, the hadronic cross section will be factorized as follows

$$
\sigma_{h p^{\uparrow} \rightarrow h J / \Psi X}(\sqrt{s})=\int d x_{\gamma} d^{2} k_{\perp \gamma} f_{\gamma / h}\left(x_{\gamma}, k_{\perp \gamma}\right) \cdot \sigma_{\gamma p^{\uparrow \rightarrow J / \Psi X}}\left(W_{\gamma p}^{2}\right),
$$

where $x_{\gamma}$ is the energy fraction of hadron carried by the photon with transverse momentum $k_{\perp \gamma}$ and $f_{\gamma / h}$ is the photon flux associated to hadron $h$. Moreover, $W_{\gamma h}$ is the c.m.s. photon-proton energy given by $W_{\gamma p}=[2 \omega \sqrt{s}]^{1 / 2}$, where $\omega$ is the photon energy and $\sqrt{s}$ is the c.m.s energy of the hadronproton system. The final state will be characterized by the presence of one rapidity gap and an intact hadron, which we assume to be the unpolarized one. Both aspects can be used in principle to experimentally separate the vector mesons produced by photon - induced interactions. In this study we will assume that the transverse momentum dependence of the photon distribution can be described by a simple Gaussian form: $f_{\gamma / h}\left(x_{\gamma}, k_{\perp \gamma}\right)=f_{\gamma / h}\left(x_{\gamma}\right) \exp \left(-k_{\perp \gamma}^{2} /\left\langle k_{\perp \gamma}^{2}\right\rangle\right) /\left(\pi\left\langle k_{\perp \gamma}^{2}\right\rangle\right)$. Moreover, the photon spectrum $f_{\gamma / h}\left(x_{\gamma}\right)$ associated to a proton is derived considering the Weizsäcker-Williams method of virtual photons and using an elastic proton form factor (For more details see Refs. $[7,8])$. In the case of $p A$ collisions, an analytic approximation for the equivalent photon flux of a 
nuclei can be calculated considering the requirement that photoproduction is not accompanied by hadronic interaction (ultra-peripheral collision) and considering a pointlike form factor.

In our analysis we will assume that the inelastic $J \Psi$ photoproduction can be described by the Color Evaporation Model (CEM) [9], generalized to take into account the transverse momentum dependence of the gluon distribution function [10]. The CEM predicts that the cross section will be given by

$$
\sigma_{\gamma p^{\uparrow} \rightarrow J / \Psi X}=F_{J / \Psi} \bar{\sigma}_{\gamma p^{\uparrow} \rightarrow c \bar{c} X}
$$

where $F\left[n J^{P C}\right]$ is process- and kinematics-independent and describes the probability that the $c \bar{c}$ pair binds to form a quarkonium of a given spin $J$, parity $P$, and charge conjugation $C$. Moreover, the short distance contribution is

$$
\bar{\sigma}_{\gamma p^{\uparrow} \rightarrow c \bar{c} X}=\int_{4 m_{c}^{2}}^{4 m_{D}^{2}} d M_{c \bar{c}}^{2} d x_{g} d^{2} k_{\perp g} f_{g / p^{\uparrow}}\left(x_{g}, k_{\perp g}\right) \frac{d \sigma[\gamma g \rightarrow c \bar{c}]}{d M_{c \bar{c}}^{2}},
$$

where $M_{c \bar{c}}$ is the invariant mass of the $c \bar{c}$ pair, $m_{c}$ is the charm quark mass and $2 m_{D}$ is the $D \bar{D}$ threshold. One have that the cross section for the inelastic $J / \Psi$ photoproduction is proportional to the number density of gluons inside a proton with transverse polarization $S$ and momentum $P$, which is usually parameterized as [11]

$$
f_{g / p^{\uparrow}}\left(x_{g}, k_{\perp g}, S\right) \equiv f_{g / p}\left(x_{g}, k_{\perp g}\right)+\frac{1}{2} \Delta^{N} f_{g / p^{\uparrow}}\left(x_{g}, k_{\perp g}\right) \hat{S} \cdot\left(\hat{P} \times \hat{k_{\perp g}}\right),
$$

where $x_{g}$ is the longitudinal momentum fraction of the gluon and $k_{\perp g}$ its transverse momentum. Moreover, $f_{g / p}\left(x_{g}, k_{\perp g}\right)$ is the unpolarized transverse momentum dependent (TMD) gluon distribution and $\Delta^{N} f_{g / p^{\uparrow}}\left(x_{g}, k_{\perp g}\right)$ is the gluon Sivers function.

In order to probe the gluon Sivers function ind the inelastic $J / \Psi$ photoproduction in $p^{\uparrow} p$ and $p^{\uparrow} A$ collisions, in what follows we will investigate the impact of different models for $\Delta^{N} f_{g / p^{\uparrow}}\left(x_{g}, k_{\perp g}\right)$ in the rapidity $(Y)$ dependence of the single spin asymmetry, defined as

$$
A_{N}(Y)=\frac{\frac{d \sigma^{\uparrow}}{d Y}-\frac{d \sigma^{\downarrow}}{d Y}}{\frac{d \sigma^{\uparrow}}{d Y}+\frac{d \sigma^{\downarrow}}{d Y}}
$$

where $\frac{d \sigma^{\uparrow}}{d Y}$ and $\frac{d \sigma^{\downarrow}}{d Y}$ are respectively the differential cross sections measured when the proton is transversely polarized up $(\uparrow)$ and down $(\downarrow)$ with respect to the scattering plane, calculated using Eqs. (2.1), (2.2) and (2.4). As in Ref. [10] we will estimate the numerator with a weight factor $\sin \left(\phi_{q_{T}}-\phi_{S}\right)$, where $\phi_{q_{T}}$ and $\phi_{S}$ are the azimuthal angles of the $J / \Psi$ and proton spin, respectively. Our motivation to investigate the rapidity dependence of $A_{N}$ is associated to the fact that the rapidity $Y$ of the vector meson determines the typical values of $x_{\gamma}$ and $x_{g}$ probed in the interaction, which are given by $x_{g, \gamma}=M_{c \bar{c}} / \sqrt{s} \exp ( \pm Y)$. Therefore, its analysis allow us to know the value of $x_{g}$ that is being probed in the gluon Sivers function. In what follows we will assume that the unpolarized TMD gluon distribution $f_{g / p}\left(x_{g}, k_{\perp g}\right)$ can be described by a Gaussian form:

$$
f_{g / p}\left(x_{g}, k_{\perp g}\right)=f_{g / p}\left(x_{g}, \mu^{2}\right) \frac{1}{\pi\left\langle k_{\perp g}^{2}\right\rangle} e^{-k_{\perp_{g}}^{2} /\left\langle k_{\perp_{g}}^{2}\right\rangle}
$$


with the factorization scale $\mu^{2}$ being given by $M_{c \bar{c}}^{2}$. As in Refs. $[12,10]$, we choose a frame where the proton is moving along the $z$-axis with momentum $P$, is transversely polarized along $y-$ axis and $k_{\perp}=k_{\perp}\left(\cos \phi_{k_{\perp, g}}, \sin \phi_{k_{\perp, g}}, 0\right)$, which implies that $\hat{S} \cdot\left(\hat{P} \times \hat{k_{\perp g}}\right)=\cos \phi_{k_{\perp, g}}$. Moreover, we will consider that the gluon Sivers function can be described as follows

$$
\Delta^{N} f_{g / p^{\uparrow}}\left(x_{g}, k_{\perp g}\right)=2 N_{g}\left(x_{g}\right) f_{g / p}\left(x_{g}, \mu^{2}\right) h\left(k_{\perp g}\right) \frac{e^{-k_{\perp_{g}}^{2} /\left\langle k_{\perp_{g}}^{2}\right\rangle}}{\pi\left\langle k_{\perp g}^{2}\right\rangle},
$$

where

$$
N_{g}\left(x_{g}\right)=N_{g} x_{g}^{\alpha}\left(1-x_{g}\right)^{\beta} \frac{(\alpha+\beta)^{(\alpha+\beta)}}{\alpha^{\alpha} \beta^{\beta}}
$$

with $\left|N_{g}\right| \leq 1$ and

$$
h\left(k_{\perp g}\right)=\sqrt{2 e} \frac{k_{\perp g}}{M^{\prime}} e^{-k_{\perp g}^{2} / M^{\prime 2}} .
$$

The $k_{\perp}$ dependent part of the Sivers function can expressed as follows

$$
h\left(k_{\perp g}\right) \frac{e^{-k_{\perp_{g}}^{2} /\left\langle k_{\perp_{g}}^{2}\right\rangle}}{\pi\left\langle k_{\perp_{g}}^{2}\right\rangle}=\frac{\sqrt{2 e}}{\pi} \sqrt{\frac{1-\rho}{\rho}} k_{\perp g} \frac{e^{-k_{\perp_{g}}^{2} / \rho\left\langle k_{\perp_{g}}^{2}\right\rangle}}{\left\langle k_{\perp_{g}}^{2}\right\rangle^{3 / 2}},
$$

where $\rho \equiv M^{\prime 2} /\left(\left\langle k_{\perp g}^{2}\right\rangle+M^{\prime 2}\right)$. The parametrization given by Eq. (2.7) was proposed in Ref. [14] and recently used in Ref. [12], where the authors have considered the midrapidity data on the transverse single spin asymmetry measured in $p p \rightarrow \pi^{0} X$ by the PHENIX Collaboration at RHIC and the present information on the quark Sivers functions to get a first estimate on the gluon Sivers distribution. Assuming $\left\langle k_{\perp g}^{2}\right\rangle=0.25 \mathrm{GeV}^{2}$, they have obtained two different sets for the best - fit parameters $N_{g}, \alpha, \beta$ and $\rho$, denoted by SIDIS1 and SIDIS2 (For details see [12]). In order to estimate the impact of different gluon Sivers distributions on $A_{N}(Y)$, we also will consider two alternative models obtained assuming that [13] (a) $N_{g}(x)=\left[N_{u}(x)+N_{d}(x)\right] / 2$ and (b) $N_{g}(x)=N_{u}(x)$, which we will denote by BV-a and BV-b hereafter. In our study we will consider the best fit parameters for the $u$ and $d$ quark Sivers functions obtained recently in Ref. [15] from the latest SIDIS data.

\section{Results}

In 1 (left panel) we present our predictions for the rapidity distribution considering $p p$ collisions at $\sqrt{s}=200$ and $500 \mathrm{GeV}$. We have assumed that $F_{J / \Psi}=(1 / 9) \cdot \rho_{J / \Psi}$, where the factor $1 / 9$ represent the statistical probability that the $c \bar{c}$ will be in a color singlet state asymptotically and $\rho_{J / \Psi}$ is a non - perturbative parameter, determined by fitting the data. We assume that $\rho_{J / \Psi}=0.5$. The rapidity distribution shown have been obtained assuming that one of the incident protons acts as the photon source and the other as target. Such assumption implies an asymmetric distribution for a symmetric collision.

Our predictions for the single spin asymmetry are presented in Fig. 1 (right panel) considering $p^{\uparrow} p$ collisions at $\sqrt{s}=200 \mathrm{GeV}$ (left panel) and $500 \mathrm{GeV}$ (right panel). We have that the magnitude 

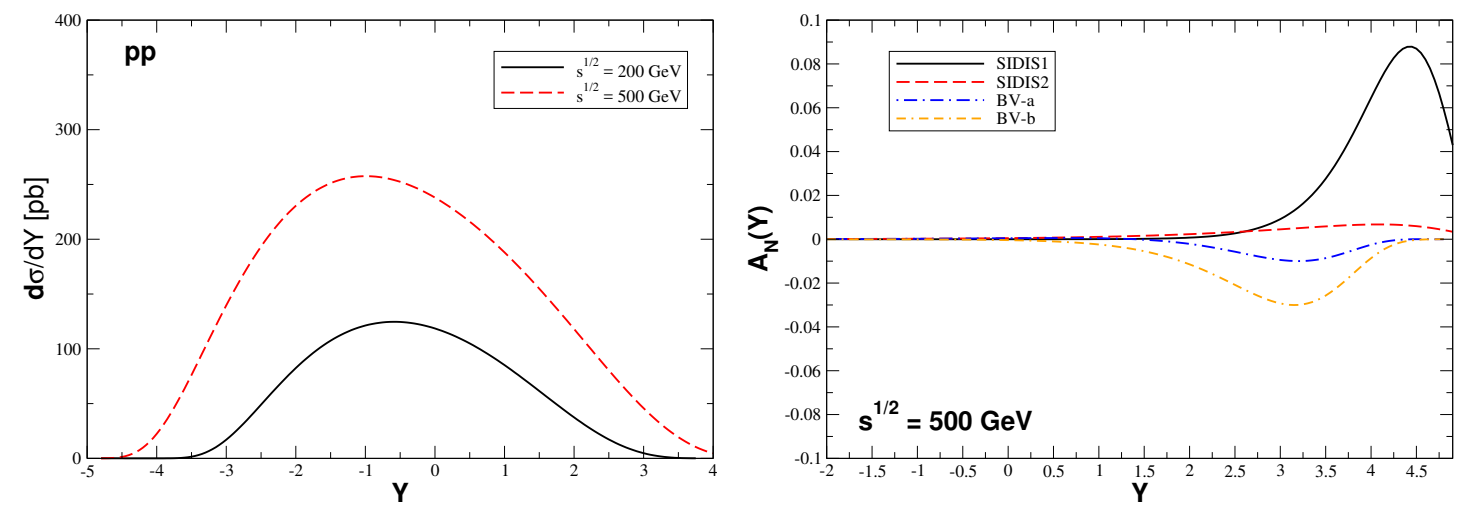

Figure 1: Dipole-nucleus scatering amplitude as a function of $r^{2}$ for fixed values of $b$ and different values of $x(A=P b)$.

and signal of $A_{N}(Y)$ is strongly dependent on the model used for the gluon Sivers function, with the position of the peak ocurring at larger values of $Y$ with the increasing of the energy. In Ref. [5] we have verified that the maximum and minimum values of $A_{N}$ are almost independent of energy. These results are consistent with those obtained in Refs. [10] for the $J / \Psi$ production in $e p^{\uparrow}$ collisions. Our results indicate that the signal and magnitude of the asymmetry can be probed by the analysis of the $J / \Psi$ production at forward rapidities. Additionally, we also have estimated $A_{N}$ for $p^{\uparrow} A u$ collisions and obtained that its rapidity dependence, position of the peak and value of the maximum and minimum are very similar to those obtained in $p^{\uparrow} p$ collisions. Such behaviour is expected, since the photon flux is present in the numerator and denominator of Eq. (2.5), which implies that the $Z^{2}$ enhancement of the nuclear flux does not affect $A_{N}$. Therefore, we predict similar asymmetry in $p^{\uparrow} p$ and $p^{\uparrow} A u$ collisions. However, it is important to emphasize that the magnitude of the rapidity distribution in nuclear collisions is almost three orders of magnitude larger than in proton - proton collisions, which implies that the study of the single spin asymmetry in $p^{\uparrow} A u$ collisions is expected to be more easily performed.

\section{Summary}

During the last years, the experimental results from Tevatron, RHIC and LHC have demonstrated that the study of hadronic physics using photon induced interactions in $p p / p A / A A$ colliders is feasible. In this contribution we have summarized the results present in Ref. [5], where we have estimated, by the first time, the inelastic $J / \Psi$ photoproduction in $p^{\uparrow} p / p^{\uparrow} A$ collisions at RHIC energies. Moreover, the impact of different models for the gluon Sivers function on the transverse single spin asymmetry have been investigated. Our results indicate that the asymmetry is strongly dependent on the modelling of the gluon Sivers function. Moreover, the signal and magnitude of the asymmetry can by investigated by the analysis of the $J / \Psi$ production at forward rapidities. Such aspects motivate a future experimental analysis of this process as a probe of the gluon Sivers function. 


\section{Acknowledgments}

This work was partially financed by the Brazilian funding agencies CNPq and FAPERGS.

\section{References}

[1] D. Boer, M. Diehl, R. Milner, R. Venugopalan, W. Vogelsang, D. Kaplan, H. Montgomery and S. Vigdor et al., arXiv:1108.1713 [nucl-th].

[2] A. Accardi, J. L. Albacete, M. Anselmino, N. Armesto, E. C. Aschenauer, A. Bacchetta, D. Boer and W. Brooks et al., Eur. Phys. J. A 52, no. 9, 268 (2016)

[3] D. W. Sivers, Phys. Rev. D 41, 83 (1990).

[4] D. Boer, C. Lorce, C. Pisano and J. Zhou, Adv. High Energy Phys. 2015, 371396 (2015)

[5] V. P. Goncalves, Phys. Rev. D 97, no. 1, 014001 (2018)

[6] G. Baur, K. Hencken, D. Trautmann, S. Sadovsky, Y. Kharlov, Phys. Rep. 364, 359 (2002); V. P. Goncalves and M. V. T. Machado, Mod. Phys. Lett. A 19, 2525 (2004); C. A. Bertulani, S. R. Klein and J. Nystrand, Ann. Rev. Nucl. Part. Sci. 55, 271 (2005); K. Hencken et al., Phys. Rept. 458, 1 (2008).

[7] M. Drees and D. Zeppenfeld, Phys. Rev. D 39, 2536 (1989).

[8] B. A. Kniehl, Phys. Lett. B 254, 267 (1991).

[9] H. Fritzsch, Phys. Lett. 67B, 217 (1977); F. Halzen, Phys. Lett. 69B, 105 (1977). F. Halzen and S. Matsuda, Phys. Rev. D 17, 1344 (1978)

[10] R. M. Godbole, A. Misra, A. Mukherjee and V. S. Rawoot, Phys. Rev. D 85, 094013 (2012); Phys. Rev. D 88, no. 1, 014029 (2013); R. M. Godbole, A. Kaushik, A. Misra and V. S. Rawoot, Phys. Rev. D 91, no. 1, 014005 (2015)

[11] M. Anselmino, U. D’Alesio and F. Murgia, Phys. Rev. D 67, 074010 (2003)

[12] U. D’Alesio, F. Murgia and C. Pisano, JHEP 1509, 119 (2015)

[13] D. Boer and W. Vogelsang, Phys. Rev. D 69, 094025 (2004)

[14] M. Anselmino, M. Boglione, U. D’Alesio, A. Kotzinian, F. Murgia and A. Prokudin, Phys. Rev. D 72, 094007 (2005) Erratum: [Phys. Rev. D 72, 099903 (2005)]

[15] M. Anselmino, M. Boglione, U. D’Alesio, F. Murgia and A. Prokudin, JHEP 1704, 046 (2017) 